\title{
LINKING AND HOLOMORPHIC HULLS
}

\author{
H. ALEXANDER
}

\section{Introduction}

If $X$ and $Y$ are disjoint compact oriented smooth submanifolds of a smooth oriented manifold $M$ and are homologous to zero in $M$, then the linking number of $X$ and $Y$, denoted $\operatorname{link}(X, Y)$ (or by $\operatorname{link}(X, Y ; M)$ for clarity) is equal to the intersection number of $V$ and $Y$, where $(V, X)$ is a compact oriented submanifold with boundary in $M$. This can be taken as one of the several equivalent definitions of linking number; here the dimensions $a, k, m$ of $X, Y$, and $M$ respectively, satisfy $a+k=m-1$. We say that $X$ and $Y$ are $\operatorname{linked}$ if $\operatorname{link}(X, Y)$ is not zero. Our object is to apply this linking notion of Gauss to the geometry of holomorphic hulls. For example, in the case that the underlying manifold $M$ is $\mathbf{C}^{n}$, our results say that the polynomially convex hull of one of the sets $X$ or $Y$ has a nonempty intersection with the other set, provided that $X$ and $Y$ are linked.

Now take $M$ to be a Stein manifold and let $X$ be a compact subset of $M$. Then the holomorphic hull of $X$ is

$$
\widehat{X}=\{p \in M:|f(p)| \leq \max \{|f(q)|: q \in X\} \text { for all } f \in A(M)\}
$$

where $A(M)$ is the space of all holomorphic functions on $M . \widehat{X}$ is a compact subset of $M$. In special cases arising from the maximum principle, $(\widehat{X}, X)$ is a smooth manifold with boundary which is foliated by complex manifolds with boundaries in $X$. In general however, $\widehat{X}$ is not so nice and may not contain any complex manifolds, or even continuous ones. Nevertheless the perception persists that the pair $(\widehat{X}, X)$ behaves like a manifold with boundary. This is the motivation for what follows. To adapt the above data on linking to this context we replace $(V, X)$ with $(\widehat{X}, X)$ where now $X$ is an arbitrary compact subset of $M$. As before $Y$ is an oriented manifold disjoint from $X$ and homologous to zero in $M$. Then, when $X$ and $Y$ are linked in an appropriate sense, the previous consequence that $V$ and $Y$ have a nonzero intersection number will be

Received July 30,1992 . The author was supported in part by a grant from the National Science Foundation. 
replaced by the cruder statement that $\widehat{X}$ and $Y$ have a nonempty intersection. To adapt the hypothesis of the manifolds $X$ and $Y$ being linked to the setting in which $X$ is an arbitrary compact set it suffices to require that $Y$ not be homologous to zero in $M \backslash X$; when $X$ is a manifold as above this is equivalent to $\operatorname{link}(X, Y)$ being nonzero.

Theorem 1. Let $M$ be a Stein manifold of (complex) dimension $n$ and $X$ a compact subset. Let $Y$ be a compact oriented submanifold of $M$ of (real) dimension $k$, disjoint from $X$, and homologous to zero in $M$. Suppose that $Y$ is not homologous to zero in $M \backslash X$. Suppose that either

(a) $0 \leq k<n-1$, or

(b) $k=n-1$ and $H^{n}(M, \mathbf{C})=0$.

Then $\widehat{X}$ has a nonempty intersection with $Y$.

Remarks. 1. Suppose that $X$ and $Y$ are now linked manifolds in $M$ of dimensions $a$ and $k$, respectively. Then, as $a+k=2 n-1$, the smaller of $a$ and $k$ is at most $n-1$. Hence the hull of the set corresponding to the smaller of $a$ and $k$ has a nonempty intersection with the other set, unless, in case (b), the smaller is $n-1$ and $H^{n}(M, \mathbf{C}) \neq 0$.

2. The cohomology condition in (b) is needed. Consider for $M$ the product in $\mathbf{C}^{n}$ of $n$ copies of $C^{*}$, the punctured plane. Let $X$ be the $n$-torus in $M$, i.e., the product of $n$ unit circles. Choose $Y$ as a $k=n-1$ sphere in $M$ disjoint from $X$ and such that $X$ and $Y$ are linked in $M$; for example, $Y$ could be a small sphere in the normal space to $X$ at some point. Then, as $\widehat{X}=X$, the intersection of $\widehat{X}$ and $Y$ is empty. Of course, $H^{n}(M, \mathbf{C}) \neq 0$.

Corollary 1. Suppose that $\mathbf{C}^{n}=S \oplus T$ is an orthogonal decomposition of $\mathbf{C}^{n}$ into real linear spaces $S$ and $T$ of real dimension $s$ and $k$ respectively with $s>n$ and let $\pi: \mathbf{C}^{n} \rightarrow S$ be the orthogonal projection to $S$. Let $E$ be a compact subset of $S$ and let $f: E \rightarrow T$ be a continuous map and let $\operatorname{Gr}(f)$ be the graph of $f$ in $\mathbf{C}^{n}$. Let $D$ be a relatively compact component of the complement of $E$ in $S$. Then $\widehat{\operatorname{Gr}(f)}$, the polynomially convex hull of $\operatorname{Gr}(f)$, covers $D$, i.e.,

$$
\pi(\widehat{\operatorname{Gr}(f)}) \supseteq D
$$

The special case of the corollary when $S$ is complex linear and $D$ is a ball appeared in [3] with two proofs and a third proof was given by Ahern and Rudin [1]. The second proof in [3], due to J.-P. Rosay, is closest to the methods of this paper. The case $n=2$ and $s=3$ where $f$ is a realvalued function on a 2-manifold is of interest. When $D$ is convex with smooth boundary, a very precise description of the hull is due to Bedford and Klingenberg [7]: the hull is a disjoint union of analytic disks. In other 
cases, the structure of the hull is less well understood, as, for example, when $D$ is a solid torus.

Another phenomenon of linking is the relationship of linking at the boundary of a domain to intersections in the domain. The prototype of such results is the following. Cf. [10, Proposition, p. 383].

Proposition. Let $(V, X)$ and $(W, Y)$ be oriented submanifolds with boundary in $\mathbf{R}^{n}$ such that $V$ and $W$ are contained in the open unit ball $B$ and such that their boundaries are contained in the unit sphere $b B$. Suppose that $X$ and $Y$ are disjoint and that $V$ and $W$ intersect transversally, if at all. Then

$$
I(V, W)=\operatorname{link}(X, Y ; b B) .
$$

Remarks. We are assuming that the linking number is defined. This means that $\operatorname{dim}(V)+\operatorname{dim}(W)=n$. Here $I(V, W)$ denotes the (signed) intersection number of $V$ and $W$. In the case that $V$ and $W$ are complex manifolds in $\mathbf{C}^{n}$ with their natural orientations, then the intersection number is just the number of points in the intersection. For example, if $V$ and $W$ are complex linear spaces of complex dimension $n$ meeting transversally at the origin in $\mathbf{C}^{2 n}$, it follows that their boundaries $X$ and $Y$, which are disjoint $2 n-1$ spheres in the boundary of the unit ball, satisfy $\operatorname{link}\left(X, Y ; S^{4 n-1}\right)=1$. With $n=1$, this fact is used in the standard computations of the Hopf invariant of the Hopf fibration (see [8, pp. 235-239]).

The following is the statement corresponding to the proposition in the case when $X$ is an arbitrary compact set in a Stein manifold and with $V$ replaced by a holomorphic hull of $X$.

Theorem 2. Let $M$ be a Stein manifold of complex dimension at least 2 , and $D$ a smoothly bounded relatively compact strictly pseudoconvex domain in $M$. Let $X$ be a compact subset of $b D$. Let $Y$ be a $k$ dimensional compact oriented smooth submanifold of bD with $0 \leq k \leq$ $n-2$ which is homologous to zero in $b D$ and which is disjoint from $X$, i.e., $Y \subseteq G: \equiv b D \backslash X$, and suppose that there is a $(k+1)$-dimensional submanifold $W$ of $D$ such that $Y=b W$. Let $\widehat{X}$ be the $\mathscr{O}_{\bar{D}}$ hull of $X$. Suppose that $Y$ links $X$ in $b D$ in the sense that $Y$ is not homologous to zero in $G$. Then $\widehat{X}$ has a nonempty intersection with $W$.

As a consequence we obtain the following corollary originally obtained by the author with E. L. Stout [4] by a different method, extending the Euclidean space case of [2]; also see [6]. The corollary was also proved by Lupaccioulu [9] who obtained more general results related to Theorem 2 in the case of pseudoconcave manifolds. Our approach is perhaps more 
geometric. With more elaborate hypotheses, the strict pseudoconvexity of $D$ in Theorem 2 could be relaxed.

Corollary 2. Let $M, D, X$ and $\widehat{X}$ be as in Theorem 2. Each component of $D \backslash \widehat{X}$ contains in its boundary exactly one component of $b D \backslash X$.

Proof. Without loss of generality we can suppose that $D$ is connected. Then $b D$ is connected, since $D$ is Stein and $n \geq 2$. It suffices to prove the following. If $p$ and $q$ are points in distinct components of $b D \backslash X$ and if $W$ is a simple smooth curve in $D$ joining $p$ to $q$, then $W$ has a nonempty intersection with $\widehat{X}$. Let $Y$ be $b W=\{q,-p\}$, a 0 dimensional submanifold of $d D$. The connectedness of $b D$ implies that $Y$ is homologous to zero in $b D$. Since $p$ and $q$ lie in different components of $b D \backslash X, Y$ is not homologous to 0 in $b D \backslash X$. Thus we can apply Theorem 2 to conclude that $\widehat{X}$ meets $W$.

\section{Proof of Corollary 1}

Set $X=\operatorname{Gr}(f)$. We argue by contradiction and suppose that there exists $p \in \pi(\widehat{X}) \backslash D$. Set $Q=\pi^{-1}(\{p\})$, a real $k$-plane in $\mathbf{C}^{n}$. Then $\widehat{X} \cap Q$ is empty. Hence $\widehat{X} \cap Y$ is empty for all geometric $k$-spheres $Y$ in $\mathbf{C}^{n}$ of sufficiently large radius $R$, which are tangent to $Q$ at $(p, 0) \in S \times T=\mathbf{C}^{n}$. It is evident and straightforward to check that $Y$ "links" $X$, i.e., $Y$ does not bound in $\mathbf{C}^{n} \backslash X$, if $R$ is sufficiently large. Since $k=2 n-s<n$, Theorem 1 implies that $\widehat{X}$ meets $Y$. Contradiction.

\section{Poincaré duals and linking}

We next recall some of the basic facts needed about Poincare duals and linking. A very nice reference for all of this is the book of Bott and Tu [8]. Our manifolds will be smooth and oriented; for such a manifold $M$ the $q$ th de Rham cohomology group will be denoted by $H^{q}(M)$, and the de Rham cohomology with compact support by $H_{c}^{q}(M)$. For a noncompact oriented manifold $M$ of dimension $m$, Poincaré duality states that

$$
H^{k}(M)=\left(H_{c}^{m-k}(M)\right)^{*}
$$

and also, if $M$ is of finite type,

$$
\left(H^{k}(M)\right)^{*}=H_{c}^{m-k}(M) .
$$


If $Y$ is a closed oriented submanifold of $M$ of dimension $k$, then its Poincaré dual is a closed $m-k$ form $\eta_{Y}$ on $M$ with the property that

$$
\int_{Y} \alpha=\int_{M} \alpha \wedge \eta_{Y}
$$

for all closed $k$ forms $\alpha$ with compact support in $M$. Sometimes to avoid ambiguity we denote the Poincare dual by $\eta_{Y}^{M}$. The form is not uniquely determined, but its cohomology class $\left[\eta_{Y}\right] \in H^{m-k}(M)$ is unique and is also referred to as the Poincare dual.

Three basic properties of the Poincare duals are:

(i) Localization. For any tubular neighborhood of $Y$ in $M$ there is a Poincaré dual $\eta_{Y}$ with support in that neighborhood.

(ii) If the oriented submanifolds $Y$ and $W$ of $M$ meet transversally, then

$$
\eta_{Y} \wedge \eta_{W}=\eta_{Y \cap W} .
$$

(iii) If $f: M^{\prime} \rightarrow M$ is an orientation-preserving map, and $Y$ is an oriented submanifold of $M$, then, assuming appropriate transversality,

$$
f^{*}\left(\eta_{Y}\right)=\eta_{f^{-1}(Y)} .
$$

In particular, if $A$ and $Y$ are oriented submanifolds of $M$ intersecting transversally, and $f$ is an inclusion map $i: A \hookrightarrow M$, then (iii) gives

$$
\left.\eta_{Y}\right|_{A}=i^{*}\left(\eta_{Y}\right)=\eta_{A \cap Y}^{A} .
$$

Let $Y$ be a compact oriented submanifold of $M$. By localization, we can take $\eta_{Y}$ with compact support in $M$. We can then ask whether $(*)$ remains valid if we drop the hypothesis that $\alpha$ have compact support in $M$. By Poincaré duality, this is so, provided that $M$ has finite type. However, even if $M$ does not have finite type, we can find a particular $\eta_{Y}$ such that

(**) $\quad \int_{Y} \alpha=\int_{M} \alpha \wedge \eta_{Y} \quad$ for all closed $k$-forms $\alpha$ on $M$.

To see this we choose a tubular neighborhood $N$ of $Y$ in $M$. Then $N$ is of finite type and so there is a "compact Poincare dual" (see [8, p. 51]) $\eta_{Y}^{\prime N}$ of $Y$ in $N$ such that

$$
\int_{Y} \beta=\int_{N} \beta \wedge \eta_{Y}^{\prime N}
$$


for all closed $k$ forms $\beta$ in $N ; \eta_{Y}^{\prime N}$ is a closed $(m-k)$-form with compact support in $N$. Now define $\eta_{Y}$ as the extension to $M$ of $\eta_{Y}^{\prime N}$ by 0 outside of $N$. Then for any closed $k$-form $\alpha$ on $M$ we have

$$
\int_{Y} \alpha=\int_{N} \alpha \wedge \eta_{Y}^{\prime N}=\int_{M} \alpha \wedge \eta_{Y}
$$

Thus (**) holds.

Suppose furthermore that $Y$ is homologous to zero in $M$ and let $\eta_{Y}$ be chosen so that $(* *)$ holds. Then we claim that $\left[\eta_{Y}\right]=0$ in $H_{c}^{m-k}(M)$. By Poincare duality it suffices to show that

$$
\int_{M} \alpha \wedge \eta_{Y}=0
$$

for all closed forms $\alpha$ on $M$. This follows from (**) because the integral over $Y$ is zero by Stokes' theorem, since $Y$ is homologous to zero in $M$. Thus there exists a $(m-k-1)$-form $\omega_{Y}$ with compact support in $M$ such that $\eta_{Y}=d \omega_{Y}$.

Suppose that $X$ and $Y$ are disjoint oriented compact submanifolds of $M$, which are homologous to zero and satisfying $s+k=m-1$ with dimensions $s$ and $k$ respectively. Then $\operatorname{link}(X, Y)$ is defined and can be computed as follows. Choose $\eta_{X}$ and $\eta_{Y}$ with compact and disjoint supports. By the last paragraph we have $\omega_{X}$ with compact support in $M$ such that $d \omega_{X}=\eta_{X}$. Then

$$
\operatorname{link}(X, Y)=\int_{M} \omega_{X} \wedge \eta_{Y}
$$

\section{Proof of Theorem 1}

We argue by contradiction and suppose that $\hat{X}$ is disjoint from $Y$. Then there exists a relatively compact $\mathscr{O}_{M}$-convex domain $\Omega$ in $M$ containing $\widehat{X}$ and such that $\bar{\Omega}$ is disjoint from $Y$. Let $\eta_{Y}$ be a Poincaré dual of $Y$ in $M \backslash X$ such that $\operatorname{spt}\left(\eta_{Y}\right)$ is disjoint from $\bar{\Omega}$ and (**) holds for $k$-forms $\alpha$ in $M \backslash X$. Extending by 0 we can view $\eta_{Y}$ as a closed form in $M$. As in $\S 3$, since $Y$ is homologous to zero in $M$, there exists a $(2 n-k-1)$-form $\omega_{Y}$ with compact support in $M$ such that $d \omega_{Y}=\eta_{Y}$. Let $D_{1}$ be a relatively compact subdomain in $M$ containing $\bar{\Omega} \cup \operatorname{spt}\left(\omega_{Y}\right)$ such that $b D_{1}$ is smooth. Choose a relatively compact subdomain $D_{2}$ of 
$\Omega$ such that $X$ is contained in $D_{2}$ and $b D_{2}$ is smooth. Set $D=D_{1} \backslash \bar{D}_{2}$. Then $\operatorname{spt}\left(\eta_{Y}\right) \subseteq D$ and $b D=b D_{1} \cup\left(-b D_{2}\right)$. As $Y$ is not homologous to zero in $M \backslash X$ there exists, by de Rham's theorem, a closed $k$-form $\alpha$ on $M \backslash X$ such that $0 \neq \int_{Y} \alpha$.

We have

$$
\begin{aligned}
0 & \neq \int_{Y} \alpha=\int_{M \backslash X} \alpha \wedge \eta_{Y} \quad(\text { by }(* *)) \\
& =\int_{D} \alpha \wedge \eta_{Y}=\int_{D} \alpha \wedge d \omega_{Y} \\
& =(-1)^{k} \int_{D} d\left(\alpha \wedge \omega_{Y}\right)=(-1)^{k} \int_{b D} \alpha \wedge \omega_{y} \quad \text { (Stokes) } \\
& =(-1)^{k} \int_{b D_{1}} \alpha \wedge \omega_{Y}+(-1)^{k} \int_{-b D_{2}} \alpha \wedge \omega_{Y} \\
& =(-1)^{k} \int_{-b D_{2}} \alpha \wedge \omega_{Y} \quad\left(\operatorname{spt}\left(\omega_{Y}\right) \cap b D_{1}=\varnothing\right)
\end{aligned}
$$

Now in case (a), $k<n-1$ and so $2 n-k-1>n$. Hence $H^{2 n-k-1}(\Omega)=0$ since $\Omega$ is Stein [5]. On $\Omega, d \omega_{Y}=\eta_{Y}=0$. Hence there is a $(n-k-2)$ form $\sigma$ on $\Omega$ such that $\omega_{Y}=d \sigma$ on $\Omega$. Thus

$$
\int_{-b D_{2}} \alpha \wedge \omega_{Y}=\int_{-b D_{2}} \alpha \wedge d \sigma=(-1)^{k} \int_{b D_{2}} d(\alpha \wedge \sigma)=0
$$

by Stokes. This contradicts the choice of $\alpha$.

In case (b), $2 n-k-1=n$. Since $(M, \Omega)$ is a Runge pair, it follows from [5] that the natural restriction map $H^{n}(M) \rightarrow H^{n}(\Omega)$ is surjective. As $H^{n}(M)=0$, we have $H^{n}(\Omega)=0$, and the argument of case (a) can be applied to arrive at the same contradiction.

\section{Proof of the Proposition}

Extend $V$ and $W$ to a neighborhood $N$ of $\bar{B}$ and choose Poincaré duals $\eta_{V}$ and $\eta_{W}$ in $N$ such that $\operatorname{spt}\left(\eta_{V}\right) \cap \operatorname{spt}\left(\eta_{W}\right)$ is a compact subset of $B$. Then $\eta_{V} \wedge \eta_{W}=\eta_{V \cap W}^{B}$ is a Poincaré dual of $V \cap W$. In particular, $\int_{B} \eta_{V \cap W}^{B}=I(V, W)$.

Let $j: b B \rightarrow N$ be the inclusion map. We may assume that $N$ is a ball. Hence there exists an $(n-k-1)$-form $\omega_{V}$ in $N$ such that $d \omega_{V}=\eta_{V}$. Set $\eta_{X}^{b B}=j^{*}\left(\eta_{V}\right)$ and $\eta_{Y}^{b B}=j^{*}\left(\eta_{W}\right)$. These are Poincaré duals on $b B$ 
with disjoint supports. Set $\omega_{X}^{b B}=j^{*}\left(\omega_{V}\right)$. Then on $b B$, we have

$$
d\left(\omega_{X}^{b B}\right)=d\left(j^{*}\left(\omega_{V}\right)\right)=j^{*}\left(d \omega_{V}\right)=j^{*}\left(\eta_{V}\right)=\eta_{X}^{b B}
$$

Thus

$$
\begin{aligned}
\operatorname{link}(X, Y ; b B) & =\int_{b B} \omega_{X}^{b B} \wedge \eta_{Y}^{b B} \\
& =\int_{b B} j^{*}\left(\omega_{V}\right) \wedge j^{*}\left(\eta_{W}\right) \\
& =\int_{b B} j^{*}\left(\omega_{V} \wedge \eta_{W}\right)=\int_{b B} \omega_{V} \wedge \eta_{W} \\
& =\int_{B} d\left(\omega_{V} \cap \eta_{W}\right) \quad(\text { Stokes }) \\
& =\int_{B} d \omega_{V} \wedge \eta_{W} \quad\left(\eta_{W} \text { is closed }\right) \\
& =\int_{B} \eta_{V} \wedge \eta_{W}=\int_{B} \eta_{V \cap W} \\
& =I(V, W) .
\end{aligned}
$$

\section{Proof of Theorem 2}

By replacing $M$ by an appropriate Stein neighborhood of $\bar{D}$ in $M$ we can assume that $(M, D)$ is a Runge pair, that $\widehat{X}$ is the $\mathscr{O}_{M}$-convex hull of $X$ and that $W$ extends to be a submanifold of $M$ which intersects $b D$ transversally in $Y$.

We argue by contradiction and suppose that $\widehat{X}$ is disjoint from $W$. Then there is a relatively compact $\mathscr{O}_{M}$ convex domain $\Omega$ containing $\hat{X}$ such that $\bar{\Omega}$ is disjoint from $W$. Let $\eta_{W}$ be a Poincaré dual on $M$ with support disjoint from $\bar{\Omega}$. Since $2 n-k-1>n$ and $M$ is Stein, $H^{2 n-k-1}(M)=0$. Hence there exists a $(2 n-k-2)$-form $\omega_{W}$ on $M$ such that $d \omega_{W}=\eta_{W} .\left(\eta_{W}\right.$ is a closed $(2 n-(k+1))$-form on $\left.M.\right)$

Let $j: G \rightarrow M$ be the inclusion map. Set $\eta_{Y}^{G}=j^{*}\left(\eta_{W}\right)$ and $\omega_{Y}^{G}=$ $j^{*}\left(\omega_{W}\right)$. Then $\eta_{Y}^{G}$ is a Poincaré of $Y$ in $G$ with compact support in $G$ such that $(* *)$ holds on $G$, at least if we choose the support of $\eta_{W}$ close to $Y$.

As $Y$ does not bound in $G$ there exists a closed $k$-form $\alpha$ on $G$ such that $\int_{Y} \alpha \neq 0$, by de Rham.

Choose a relatively compact domain $E_{1}$ of $\Omega \cap b D$ such that $b E_{1}$ is smooth and $X \subseteq E_{1}$. Set $E=b D \backslash E_{1}$. Then $Y \subseteq E \subseteq G$ and 
$b E=-b E_{1} \subseteq \Omega \cap G$. Thus we have

$$
\begin{gathered}
0 \neq \int_{Y} \alpha=\int_{E} \alpha \wedge \eta_{Y}^{G} \quad\left(\text { by }(* *) ; \operatorname{spt}\left(\eta_{Y}^{G}\right) \subseteq E\right) \\
=\int_{E} \alpha \wedge d \omega_{Y}^{G}=(-1)^{k} \int_{E} d\left(\alpha \wedge \omega_{Y}^{G}\right) \\
=(-1)^{k} \int_{b E} \alpha \wedge \omega_{Y}^{G} \quad \text { (Stokes). }
\end{gathered}
$$

We now consider two cases. First suppose $k<n-2$. Then $2 n-k-2>n$ and therefore $H^{2 n-k-2}(\Omega)=0$, as $\Omega$ is Stein. Since $d \omega_{Y}=\eta_{Y}=0$ on $\Omega$, there exists a $(2 n-k-3)$-form $\sigma$ on $\Omega$ such that $d \sigma=\omega_{Y}$ on $\Omega$. Set the inclusion map $i: \Omega \cap b D \rightarrow \Omega$ and set $\sigma^{\prime}=i^{*}(\sigma)$. Then, on $b E$, $d \sigma^{\prime}=i^{*}\left(\omega_{Y}\right)=\omega_{Y}^{G}$ and so

$$
\begin{aligned}
\int_{b E} \alpha \wedge \omega_{Y}^{G} & =\int_{b E} \alpha \wedge d \sigma^{\prime} \\
& =(-1)^{k} \int_{b E} d\left(\alpha \wedge \sigma^{\prime}\right)=0 \quad \text { (Stokes), }
\end{aligned}
$$

this contradicts the choice of $\alpha$.

In the second case $k=n-2$ and $2 n-k-2=n$. Since $(M, \Omega)$ is a Runge pair, the natural restriction $H^{n}(M) \rightarrow H^{n}(\Omega)$ is surjective [5]. Since $\omega_{W}$ is closed on $\Omega$, we conclude there exists a closed $n$-form $\phi$ on $M$ and an $(n-1)$-form $\theta$ on $\Omega$ such that

$$
\omega_{W}=\phi+d \theta
$$

on $\Omega$. Hence

$$
\begin{aligned}
\int_{b E} \alpha \wedge \omega_{Y}^{G} & =\int_{b E} \alpha \wedge \phi+\int_{b E} \alpha \wedge d \theta \\
& =\int_{E} d(\alpha \wedge \phi)+(-1)^{k} \int_{b E} d(\alpha \wedge \theta)
\end{aligned}
$$

by Stokes' theorem. Again by Stokes the last integral vanishes. Also the integral over $E$ vanishes since $\alpha \wedge \phi$ is closed because $\alpha$ and $\phi$ are closed (and defined on $E$ ). This again contradicts the choice of $\alpha$ and completes the proof.

\section{References}

[1] P. Ahern \& W. Rudin, Hulls of 3-spheres in $C^{3}$, Contemporary Math., Vol. 137, Amer. Math. Soc., Providence, RI, 1992, 1-28.

[2] H. Alexander, A note on polynomial hulls, Proc. Amer. Math. Soc. 33 (1972) 389-391.

[3] __, Polynomial hulls of graphs, Pacific J. Math. 147 (1991) 201-212.

[4] H. Alexander \& E. L. Stout, A note on hulls, Bull. London Math. Soc. 22 (1990) 258-260. 
[5] A. Andreotti \& R. Narasimhan, A topological property of Runge pairs, Ann. of Math. (2) 76 (1962) 499-509.

[6] R. F. Basener, Complementary components of polynomials hulls, Proc. Amer. Math. Math. Soc. 69 (1978) 230-232.

[7] E. Bedford \& W. Klingenberg, On the envelope of holomorphy of a 2-sphere in $C^{2}$, $\mathrm{J}$. Amer. Math. Soc. 4 (1991) 623-646.

[8] R. Bott \& L. Tu, Differential forms in algebraic topology, Graduate Texts in Math., Vol. 52, Springer, New York, 1982.

[9] G. Lupacciolu, Topological properties of q-convex sets, Trans. Amer. Math. Soc., to appear.

[10] W. Fulton, Intersection theory Ergeb. Math. Grenzgeb., Vol. 2, Springer, New York, 1984.

UNiversity of ILlinois at Chicago 